\title{
SYNTHESIS OF AN INTELLIGENT UAV CONTROL SYSTEM BASED ON FUZZY LOGIC IN EXTERNAL DISTURBANCE CONDITIONS
}

Submitted: $20^{\text {th }}$ January 2020; accepted: $31^{\text {st }}$ July 2020

\author{
Igor Korobiichuk, Dmytro Shevchuk, Iryna Prokhorenko, \\ Nataliia Tymoshenko, Yaroslav Smityuh, Regina Boyko
}

DOI: 10.14313/JAMRIS/3-2020/26

\begin{abstract}
To ensure reliable execution of flight tasks in the presence of both external perturbations and internal parametric perturbations, deterioration of the characteristics of the sensors, a control system structure based on intelligent technologies is proposed. The process of forming a "knowledge base" of a fuzzy controller is considered. The results of mathematical modeling of the longitudinal UAV control channel with a PID-controller and a fuzzy controller in the control loop are presented.
\end{abstract}

Keywords: Unmanned Aerial Vehicle (UAV), Fuzzy controller, Longitudinal control channel, Height stabilization, Automatic control system, Pilot-navigation complex

\section{Introduction}

The modern development of society requires the use of UAVs to solve a wide range of problems of varying complexity, which requires the improvement and creation of new UAVs, as well as their pilot-navigation complexes with the use of intellectual technologies. UAVs are extremely relevant today, trusting the investigation of large detainees found with the safety and life of people [1]. Unmanned aerial vehicles do not require any pilot on board and can be operated autonomously or remotely operated by the pilot $[2,3]$.

For today information technologies have radically changed the concept of UAVs and expanded their military and civilian use $[4,5]$. Drones are indispensable in solving of a number of civilian tasks, such as: finding, detecting and identifying objects; disaster monitoring and control [6]; monitoring of oil and gas pipelines; fire detection [7]; search and rescue [8]; observing of public events [9]; observation of land and sea traffic [10]; ecological control and monitoring of plant growing [11]; terrestrial display and photographing [12]; meteorological observation; transportation of cargo; aero-photography; traffic monitoring and control $[13,14]$.

UAVs are indispensable during military missions. Thus, for the promising UAVs, it is possible to outline such basic tasks as: reconnaissance of above-ground, air and naval targets, terrain exploration; radiation, chemical and biological investigation; installation of radio interference; fire management and targeting of ground, air and marine firearms; evaluation of the results of blows on the enemy $[15,16]$.

It should be mentioned that the development of UAVs for various purposes requires a number of topical tasks, including the development of an onboard control system and flight stabilization in the conditions of external disturbances $[17,18]$, as well as onboard high-precision navigation system. For example, a UAV flight control system must satisfy a number of conflicting requirements: reliability, simplicity of design, low cost, light weight and power consumption of actuators on the one hand, on the other hand: the accuracy of flight control in the conditions of external UAV perturbations. A compromise between different options can be achieved by use in the production of onboard UAV control system of modern intelligent control methods $[19,20]$ : artificial neural [21]; fuzzy logic [22]; genetic algorithms. As follows, the relevance of the above studies is to develop an onboard UAV control system based on modern intellectual technologies, which will improve the quality and accuracy of stabilization of its motion parameters in the conditions of external disturbances.

\section{Materials and Methods of Research}

In Fig. 1. Typical UAVs are presented which were developed at the National Aviation University (Ukraine, Kyiv) [23]. Those UAV's are physically constructions [23]. As a typical UAV is considered M-7D "Heavenly patrol" (Fig. 2d). This UAV is a twin-engine aircraft of the normal scheme with a high wing. There is an opening under the gondola for mounting the bottom / front camera. Main UAV technical characteristics: starting mass, kg - up to 150; payload weight, $\mathrm{kg}$ - up to 50; top speed, km / h. - 190; maximum flight altitude, $\mathrm{m}$ - up to 5000; maximum flight duration, h. - up to 10; method of start and landing - by plane; control modes - automatic / semi-automatic. UAVs can be used for patrolling linear objects, mapping and aerial photography, real-time video surveillance, etc. [23]. The numerical indicators for the control system with actuators of the M-7D "Heavenly patrol"[23] is $2 \mathrm{~kg}$. 


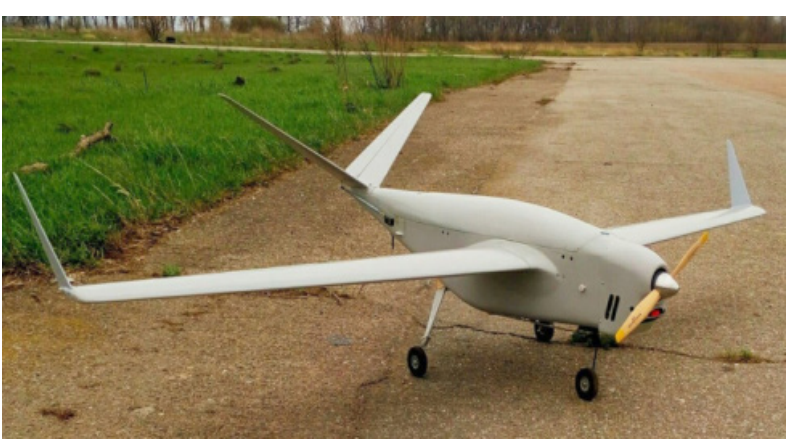

a)

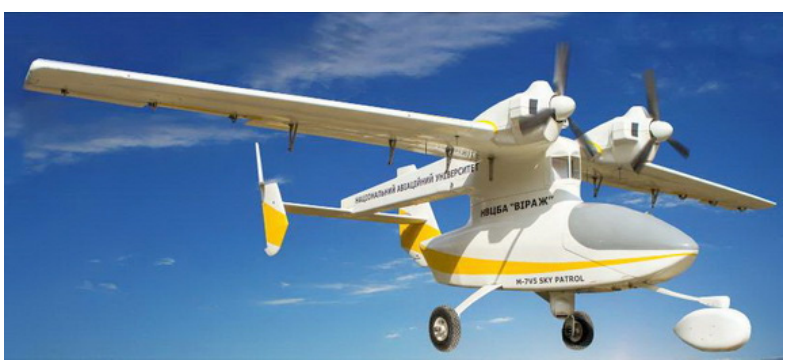

b)

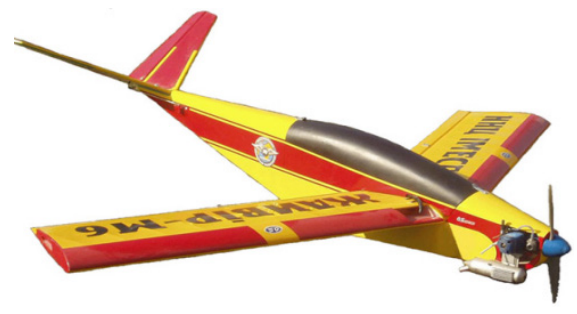

c)

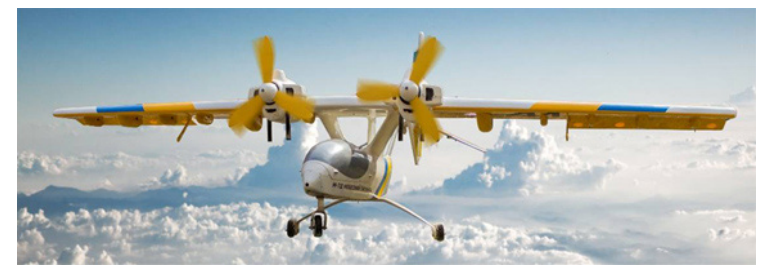

d)

Fig. 1. Typical UAVs which were developed in NAU: a) M-6-3 "Zhaivir"; b) M-7V5 "Heavenly patrol"; c) BTS M-10 "Eye 2"; d) M-7D "Heavenly patrol"

Structural and functional diagram of the pilot-navigation complex M-7D "Heavenly patrol" with the use of intellectual technologies is presented in the following form in Fig. 2.

The UAV pilot-navigation complex is structured as three modules of main units (trajectory control system, intelligent flight control system and navigation system), which are connected by cables. The UAV navigation system consists of a satellite navigation system (SNS), which operates offline and is designed to determine the location and speed of UAV flight. SNS receiver - is a combined module of GPS receiver and antenna. Performs UAV coordinates and transmits current information to the navigation system. The satellite navigation system provides information in a geocentric coordinate system linked to the center of the
Earth. But used system is a topographic coordinate system, so it is necessary to translate the information into a topographic system. The air-data computer (ADC) includes static pressure sensor (SPS) and full pressure sensor (FPS) and an ambient temperature sensor (ATS). The trajectory of the control system consists of a coordinate converter (R - UAV's position vector; $\mathrm{V}$ - airspeed), a trajectory control system and a flight path setter that stores points of space (altitude, latitude, and longitude in the geographical coordinate system).

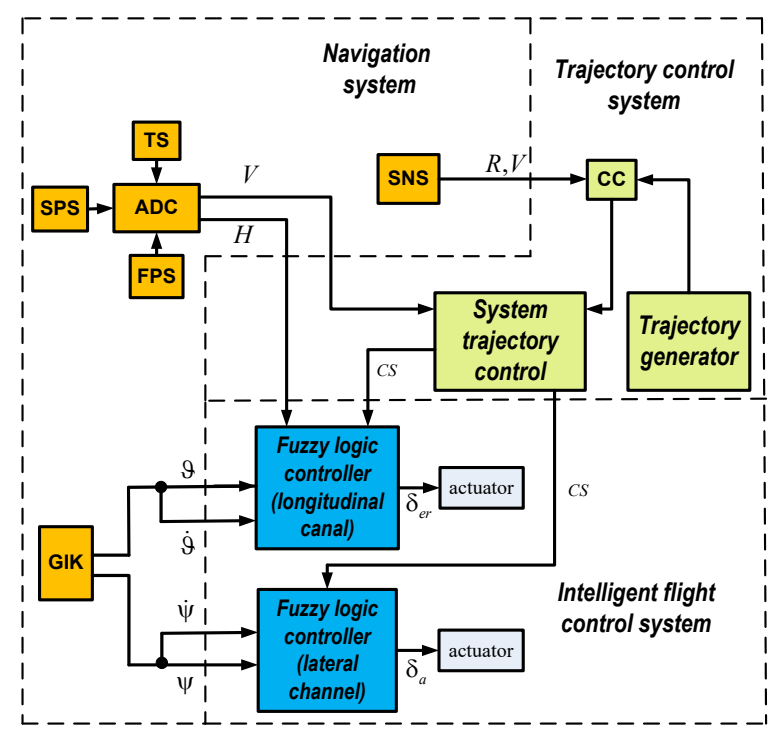

Fig. 1. Structure of the UAV M-7D Heavenly Patrol PilotNavigation Complex Using Intelligent Technologies, where: FPS - full pressure sensor; SPS - static pressure sensor; TS - temperature sensor; ADC - air-data computer; GIC - gyro-induction compass; SNS - satellite navigation system; CC - coordinate converter ( $\mathrm{R}$ - plane position vector; $\mathrm{V}$ - airspeed);intelligent flight control system; height $\mathrm{H}$; CS - command signal $\psi$, $\vartheta-$ yaw angle and pitch angle

The intelligent flight control system consists of two main modules: fuzzy UAV longitudinal motion controller and fuzzy UAV lateral motion controller. Fuzzy controllers take data from the navigation system and produce control effects based on bases of laws of management in the form "IF (flight situation) THEN (required control effect)", and then submit them in the form of signals to the UAVs (steering heights and ailerons). So, the longitudinal control channel is presented as: height $\mathrm{H}$, pitch angle $\vartheta$ and its derivative $\dot{\vartheta}$, cs - a command signal that switches the mode circuit. For the longitudinal control channel, these are modes such as dial mode and height stabilization. In the presented UAV model, the control plane - elevation rudder $\delta_{e r}$ (Fig. 2). Side angle feed: angle of lightning $\psi$ and its derivative $\psi$; cs a command signal that switches the mode circuit. For the lateral control channel, these are modes such as reversal mode, setpoint mode, setpoint stabilization mode. In the presented UAV model, the control 
plane - ailerons $\delta_{a}$ (Fig. 2). In order to ensure the competitiveness and efficiency of the use of UAVs, it is necessary for them to have a low cost and large weight of useful weight.For the purpose to provide these requirements a minimum number of low-cost sensors are set on board of the UAV, which means that the available measurements contain noise, which makes the synthesis of an efficient UAV motion control system much more difficult, and the use of standard control laws becomes impossible or inefficient. Taking into account all these features, as well as to avoid the use of slow-acting expensive adaptive systems, there is a need to synthesize control systems based on intelligent technologies that allow maintaining the controllability and stability of UAVs in the conditions of external disturbances.

Consider forming a "base of laws" of control in a fuzzy UAV controller. Let's represent UAV as an object of control with $m_{n}$ inputs and one output which corresponds to the controlling influence on the executive bodies:

$$
p=f\left(m_{1}, m_{2}, \ldots m_{n}\right)
$$

where: $f$ - output variable (angle of deflection of rudder of height, direction, ailerons, etc.); $m_{1}, m_{2}, \ldots m_{n}$ - input variables (speed, height, angles of attack, sliding, pitch, roll, yaw, it derivatives etc).

Variables $m_{1}, m_{2}, \ldots m_{n}$ and $p$ are quantitative, so the known limits of their change are assumed:

$$
\begin{gathered}
M_{i}=\left[m_{i \min }, m_{i \max }\right], i=\overline{1, n} ; \\
P=\left[p_{\min }, p_{\max }\right],
\end{gathered}
$$

where: $m_{i \min }\left(m_{i \max }\right)$ - the minimum (maximum) value of the input variable $m_{i}, i=\overline{1, n} ; P=\left[p_{\min }, p_{\max }\right]-$ the minimum (maximum) value of the output variable $p$.

The task is to vector $M^{*}=\left[m_{1}{ }^{*}, m_{2}{ }^{*}, \ldots, m_{n}{ }^{*}\right]$ of fixed values of input variables $m_{i}^{*} \in M_{i}, i=\overline{1, n}$ UAV to determine the required action of the automatic control system with a fuzzy controller $p^{*} \in P$, that is, a clear value of the regulated value is submitted to the UAV executive bodies.

A necessary condition for the formal solution of such problem is the presence of dependency (1). For example, to determine this dependency in a longitudinal channel UAV control, we'll consider the input variables $x_{i} \in X=\left[V, \alpha, H, \vartheta, \omega_{z}\right]^{\mathrm{T}}, i=\overline{1,6}$, where $V$ - flight speed, $\alpha$ - angle of attack, $H$ - flight altitude, $\vartheta$ - pitch angle, $\omega_{Z}$ - the angular velocity of change of the pitch angle and the output variable $p=\delta_{e r}$, where $\delta_{e r}$ - rudder as linguistic variables given by universal sets (2) (3)

To evaluate linguistic variables $m_{i}, i=\overline{1, n}$ i $p$ we'll use fuzzy sets: $S_{i}=\left[s_{i}^{1}, s_{i}^{2}, \ldots, s_{i}^{l_{i}}\right]-$ fuzzy variable set $m_{i}$, $i=\overline{1, n} ; V=\left[v_{1}, v_{2}, \ldots v_{r}\right]$ - fuzzy variable set $p$, where $s_{i}^{q}-q-$ is a fuzzy set of linguistic variables $m_{i}$, $q=\overline{1, l_{i}}, i=\overline{1, n} ; v_{j}-j-$ is a fuzzy set of linguistic variables $p ; r$ - number of different solutions in this field. In general case $l_{1} \neq l_{2} \neq \ldots \neq l_{n}$.

Names of individual terms $s_{i}^{1}, s_{i}^{2}, \ldots, s_{i}^{l_{i}} S_{i}{ }^{1}, s_{i}{ }^{2}, \ldots, s_{i}^{\mathrm{li}}$ may also differ from each other for different linguistic variables $m_{i}, i=\overline{1, n}$. Linguistic sets $s_{i}^{q} \in S_{i}$ i $v_{j} \in V$ $q=\overline{1, l_{i}}, i=\overline{1, n}, j=\overline{1, r}$ we'll consider fuzzy sets given by universal sets $M_{i}$ and $P$, defined by the ratios (2) (3).

Fuzzy sets $s_{i}^{q}$ and $v_{j}$ we'll define the ratios:

$$
\begin{gathered}
s_{i}^{q}=\int_{m_{i \min }}^{m_{\text {max }}} \mu^{q} s_{i}^{q}\left(m_{i}\right) / m_{i} ; \\
v_{j}=\int_{p_{\min }}^{p_{\max }} \mu^{v_{j}}(p) / p,
\end{gathered}
$$

where: $s_{i}^{q}\left(m_{i}\right)$ - function of belonging of the input variable $m_{i} \in\left[m_{i \min }, m_{i \max }\right]$ to the set $s_{i}^{q} \in S_{i}, q=\overline{1, l_{i}}, i=\overline{1, n}$; $\mu^{v_{j}}(y)$ - function of belonging of the output variable $p \in\left[p_{\text {min }} p_{\text {max }}\right]$ - to the solution $v_{j} \in V, j=\overline{1, r}$.

The available expert data, presented in the form of a knowledge matrix, establishes a connection between the set of input parameters which characterize the current state of the UAV $m_{1}-m_{n}$ and appropriate management influences $v_{j} j=\overline{1, r}$ to UAV's executive bodies, in the form of logical statements of the type "IF (flight situation) THEN (control influence required)" in the following form:

$$
\bigcup_{q=1}^{k_{j}}\left[\bigcap_{i=1}^{n}\left(m_{i}=s_{i}^{j q}\right)\right] \rightarrow p=v_{j}, j=\overline{1, r} .
$$

where: $\cup$ (or), $\cap$ (and); $v_{j}(j=\overline{1, r})$ - linguistic evaluation of the output variable $p$, determined from a fuzzy set $P ; s_{i}^{j q}$ - linguistic evaluation of the input variable $m_{i}$ in $q$-th row of the $j$-th disjunction selected from the corresponding fuzzy set $S_{i}, i=\overline{1, n}, j=\overline{1, r}, q=\overline{1, k_{j}} ; k_{j}-$ the number of rules that determine the value of the output variable of the controller on the UAV actuators.

In conclusion, to resolve the issue of formalizing the process of making a managerial existence based on fuzzy logic, note the following:first, the representation of the input parameters (altitude and flight speed, pitch angle, etc) of the fuzzy UAV controller in the form of linguistic variables with fuzzy sets (negative large, negative small, zero, positive large, and so on) allows to describe the cause-effect relationships "input parameters - control effects" in natural language using fuzzy logical statements.

\section{The Results of Research}

Analysis of the dynamic characteristics of UAV, allows us to formulate the basic requirements for flight control system (FCS) in the following form:

- FCS should provide an acceptable quality of transients under the influence of deterministic control signals, as well as stochastic disturbances of the environment (horizontal and vertical gusts of wind).For example, for a typical UAV (Fig. 1.d), the time for the establishment of a transient process by a command to increase (decrease) the height by $50 \mathrm{~m}$ should be no more than 10 seconds, overshoot $\%$, the oscillations should be damped in no more than 1 period. 


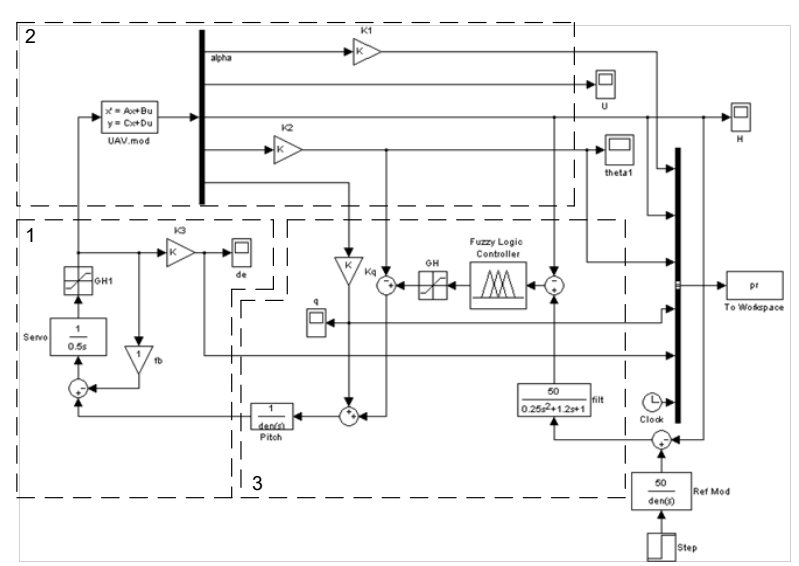

Fig. 3. Structural scheme of modeling of longitudinal motion of UAV in MATLAB environment, where: block 1 - UAV drive model; block 2 - UAV longitudinal motion model represented in state space; block 3 - UAV autopilot model

- to ensure the necessary level of adaptability of FSC, these requirements must be maintained for parametric disturbed UAV motion models. In addition, it is also necessary to further reduce the region of uncertainty associated with the influence of the Reynold's number on the stability of aerodynamic parameters.

Thus, FSC must satisfy such conflicting requirements as minimizing the UAV motion stabilization error along given flight path under the action of deterministic and stochastic external disturbances and ensuring the adaptability of the system with respect to internal parametric disturbances or in the presence of uncertainty in the parameters of the UAV mathematical model. All this can be achieved by using an autopilot based on intelligent technologies in the UAV control loop.

Let us assume, that investigated UAV, will be described in the space of states of the following equation:

$$
\begin{aligned}
& \dot{x}=\mathbf{A x}+\mathbf{B u}+w ; \\
& y=C x+v ;
\end{aligned}
$$

where: $\mathrm{x}$ - state vector; $\mathbf{A}, \mathbf{B}$ - state and control matrix; $\mathrm{u}$ - vector of control effects; $\mathrm{y}$-observation vector; C - observation matrix; $\mathrm{w}, \mathrm{v}$ - state noises and observations.

The vector of measured coordinates in longitudinal motion has the following form:

$$
\mathrm{y}=\left[h, \vartheta, \omega_{Z}\right]^{\mathrm{T}} .
$$

The vector of the UAV state in longitudinal motion in the state space is represented in the following form:

$$
x=\left[V, \alpha, \vartheta, \omega_{Z}, h\right]^{\mathrm{T}},
$$

where: $V$ - actual airspeed (AAS); $\alpha$ - angle of attack, $\vartheta$ - pitch angle; $\omega_{Z}$ - relative angular velocity; $h$ - UAV flight altitude.

The control vector in longitudinal motion this is the deflection of the steering wheel height, namely:

$$
u=\left[\delta_{e r}\right]^{\mathrm{T}}
$$

where: $u$ - control vector; $\delta_{e r}$ - elevation rudder.

In Fig. 3 is a structural diagram of UAV motion simulation in a longitudinal control channel.

For example, let's depict the fuzzy logic condoler (longitudinal canal) with two input variables $\left(x_{1}, x_{2}\right)$. For the variable $x_{1} 7$ terms are used, for the variable $x_{2}-7$ terms, for the variable $x_{3}-7$ terms (Fig. 4.).

An example of such a network can be a system consisting of the following types of rules:

Example1: IF $x_{1}$ is $N B$ AND $x_{2}$ is $N B$ THEN $y$ is $N B$,

Example 2: IF $x_{1}$ is $N B$ AND $x_{2}$ is $N M$ THEN $y$ is $N M$,

Example 3: IF $x_{1}$ is $N M$ AND $x_{2}$ is $N S$ THEN $y$ is $N M$,

where: $x_{1}, x_{2}$, - input variables, $y$ - outputvariable, $N B$ (negative big), NS (negative medium), NS (negative small) certain sets with membership functions of the triangle type.

Analyzing the main architecture of the fuzzy logic controller its generalized version can be seen (Fig. 4.).

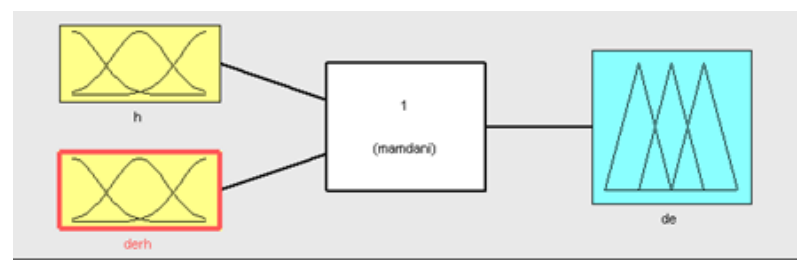

Fig. 4. Generalized architecture of a neuro-fuzzy system with a logical conclusion subsystems

Fragment of the database of a fuzzy autopilot (block 3 of Fig. 3) in the form of "IF...THEN" of the control rules connecting two input variables "height mismatch error" and "rate of change of error mismatch in height" with the output variable "angle of rotation of steering wheel height" is presented in Fig. 5.
Fig. 5. Display of a fragment of the "knowledge base" of the fuzzy controller in the form of "IF ... THEN" control rules 
The UAV control surface is shown in Fig. 6 which connects two input parameters (height mismatch error and rate of change of error mismatch in height) with the output parameter (angle of rotation of steering wheel height).

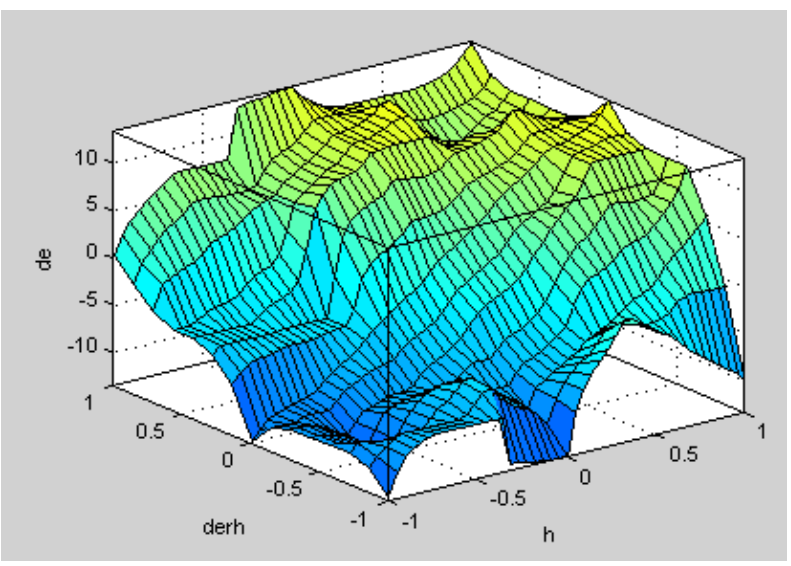

Fig. 6. UAV control surface connecting input and output variable

The process of processing the "database" of the fuzzy controller and the formation of the control action on the elevator is shown in Fig. 7.

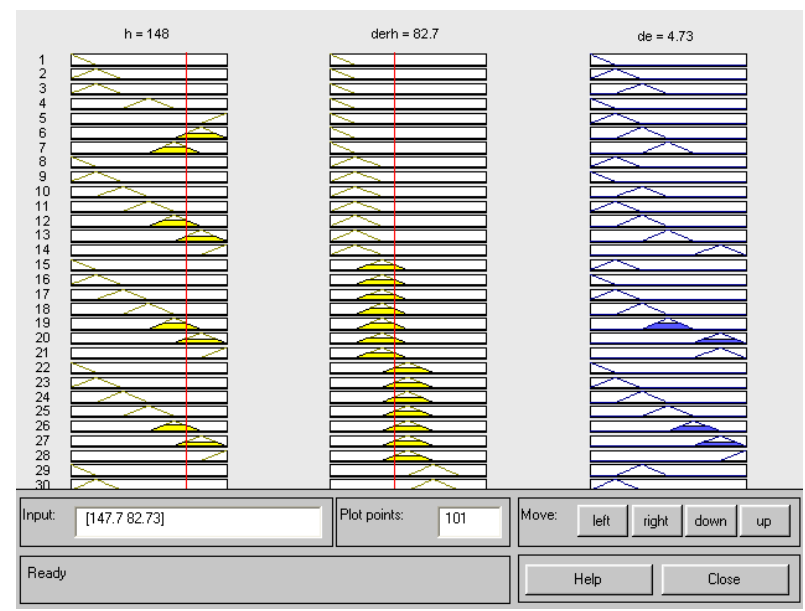

Fig. 7. The process of processing the "database" of the fuzzy controller

In Fig. 8 the results of mathematical modeling of UAV longitudinal motion control is presented.In the simulation process the PID-controller is compared with the control law $u=k_{1}+k_{2} / p+k_{3} p$, where $k_{1}=0,12$, $k_{2}=2,1, k_{3}=3,45$ - gain factors, as well as autopilot with a base of "control laws" (Fig. 5).

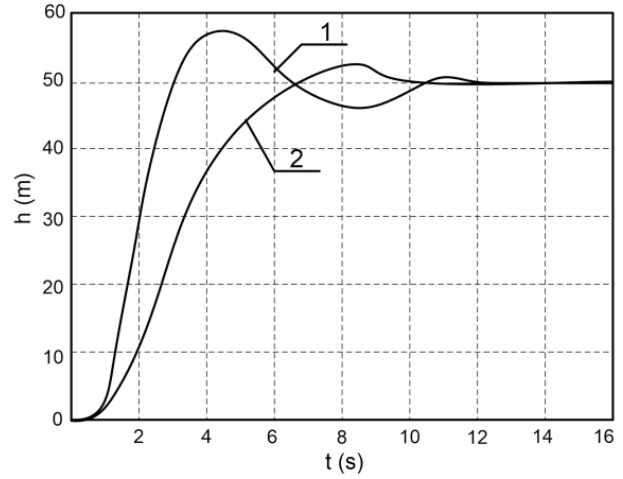

a)

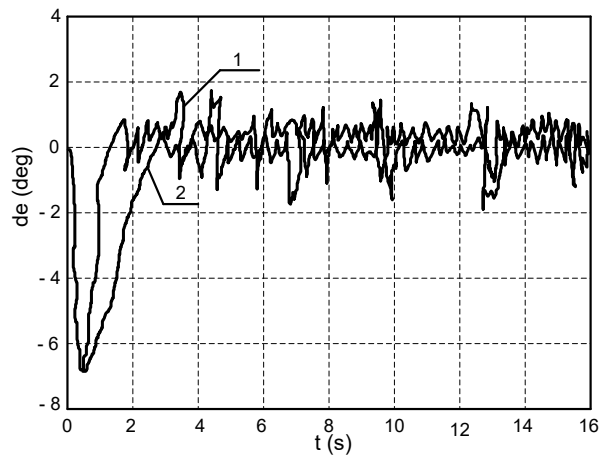

b)

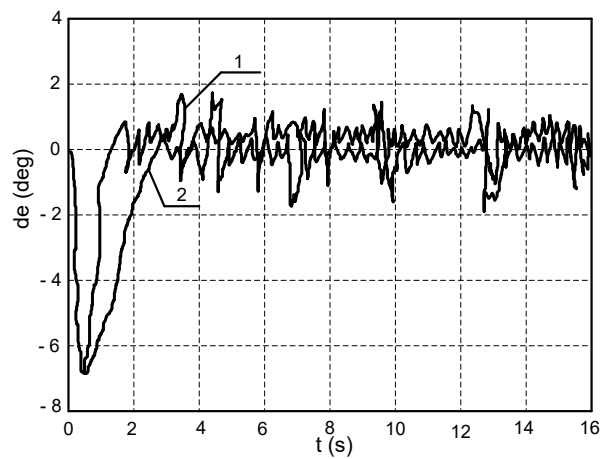

c)

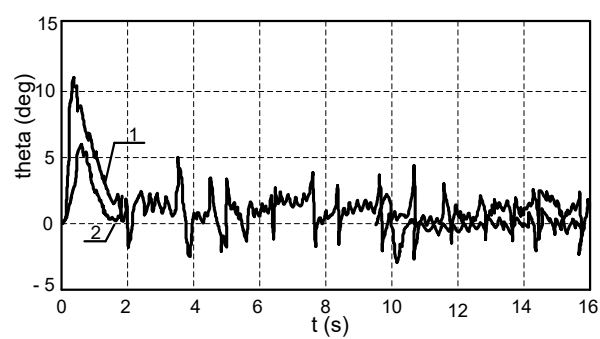

d)

Fig. 8. Transient management in longitudinal motionwhere:1 - PID-controller, 2 - fuzzy controller: a) by height; b) by the angular velocity of the pitch; c) by an elevation steering angle; d) by the pitch angle 
A comparative analysis of the results shows that the control loop of a fuzzy controller with a base of "control laws" is used it provides an increase in the of the speed of stabilization of altitude in comparison with the PID-controller under the conditions of wind gust $10 \mathrm{~m} / \mathrm{s}$ by $15 \%$, and also reduces energy costs on the steering wheel deviation by $18 \%$.

Controllers Schneider M340 or Schneider M540 and Matlab software can be used for implementing this intelligent control system based on fuzzy logic.

The results mathematical modelling can not be applied to other classes of UAVs, because in mathematical model used specific values for UAV M-7D Heavenly Patrol

\section{Conclusion}

For the UAV class which is considered, an important task is reducing of the number of sensors which is used if to take into account the reduce of cost of production. By reducing the number of sensors, it is possible to reduce the cost of both the control system and the UAV in general, which in turn makes the synthesized control system based on intelligent technologies accessible to a wider range of users with the desired characteristics of transients. Also, it should be noted that in the conventional methods of UAV control under uncertainty, the generalized control rule is set by a single law, and the fuzzy control uses a large number of partial laws. Each law operates in a given area of information space, which reflects an aerodynamic properties of this type of UAV, as well as the change of the external environment, which allows to provide the properties of adaptation to deterministic and stochastic external perturbations.

\section{AUTHORS}

Igor Korobiichuk* - ŁUKASIEWICZ Research Network - Industrial Research Institute for Automation and Measurements PIAP, Jerozolimskie 202, 02-486 Warsaw, Poland,

e-mail: igor.korobiichuk@piap.lukasiewicz.gov.pl.

Dmytro Shevchuk - National Aviation University, Lyubomyr Huzar Avenue, 1, 03058, Kyiv, Ukraine, e-mail: dmitroshevchuk@gmail.com.

Iryna Prokhorenko - National Aviation University, Lyubomyr Huzar Avenue, 1, 03058, Kyiv, Ukraine, e-mail: i.prokhorenko@nau.edu.ua.

Nataliia Tymoshenko - National Aviation University, Lyubomyr Huzar Avenue, 1, 03058, Kyiv, Ukraine, e-mail: n.tymoshenko@nau.edu.ua.

Yaroslav Smityuh - Ukrainian State University of Food Technologies, 68 Volodymyrska Street, 01033, Kyiv, Ukraine,

e-mail: Smityuh1@gmail.com.
Regina Boyko - Ukrainian State University of Food Technologies, 68 Volodymyrska Street, 01033, Kyiv, Ukraine,

e-mail: rela@ukr.net.

*Corresponding author

\section{REFERENCES}

[1] R. Austin, Unmanned Aircraft Systems: UAV Design, Development and Deployment, WileyBlackwell, 2010.

[2] J. Bishop, "The Role of Affective Computing for Improving Situation Awareness in Unmanned Aerial Vehicle Operations: A US Perspective". In: J. Vallverdú (eds.), Handbook of Research on Synthesizing Human Emotion in Intelligent Systems and Robotics, 2015, 10.4018/978-1-4666-7278-9.ch020.

[3] "Cir 328 AN/190 Unmanned Aircraft Systems (UAS)".InternationalCivilAviation Organization, https://www.icao.int/Meetings/UAS/Docu ments/Circular\%20328_en.pdf. Accessed on: 2020-12-10.

[4] I. Korobiichuk, Y. Danik, O. Samchyshyn, S. Dupelich and M. Kachniarz, "The estimation algorithm of operative capabilities of complex countermeasures to resist UAVs", SIMULATION, vol. 95 , no. $6,2019,569-573$ 10.1177/0037549718791264.

[5] I. Korobiichuk, M. Nowicki, Y. G. Danik, S. Dupelich and S. Oleksyj, "The Selection Methods for Multisensor System Elements of Drone Detection". In: R. Szewczyk and M. Kaliczyńska (eds.), Recent Advances in Systems, Control and Information Technology, 2017, 20-26, 10.1007/978-3-319-48923-0_3.

[6] H. Choi, M. Geeves, B. Alsalam and F. Gonzalez, "Open source computer-vision based guidance system for UAVs on-board decision making". In: 2016 IEEE Aerospace Conference, 2016, 10.1109/AERO.2016.7500600.

[7] M. Chen, Q. Hu, C. Mackin, J. F. Fisac and C. J. Tomlin, "Safe platooning of unmanned aerial vehicles via reachability". In: 2015 54th IEEE Conference on Decision and Control (CDC), 2015, 4695-4701, 10.1109/CDC.2015.7402951.

[8] N. Rupasinghe, A. S. Ibrahim and I. Guvenc, "Optimum Hovering Locations with Angular Domain User Separation for Cooperative UAV Networks". In: 2016 IEEE Global Communications Conference (GLOBECOM), 2016, 10.1109/GLOCOM.2016.7842113.

[9] M. Naphade, G. Banavar, C. Harrison, J. Paraszczak and R. Morris, "Smarter Cities and Their Innovation Challenges", Computer, vol. 44, no. 6, 2011, 32-39,

10.1109/MC.2011.187.

[10] T. J. Zajkowski, "Unmanned aerial vehicles: Remote sensing technology for the USDA For- 
est Service", Project Report RSAC-1507-RPT1, Remote Sens. Application Center, Salt Lake City, Utah, 2003.

[11] C. Brodbeck, E. Sikora, D. Delaney, G. Pate and J. Johnson, "Using Unmanned Aircraft Systems for Early Detection of Soybean Diseases", Advances in Animal Biosciences, vol. 8, no. 2, 2017, 802-806, $10.1017 /$ S2040470017001315.

[12] U. E. Franke, "Civilian Drones: Fixing an Image Problem?", https://isnblog.ethz.ch/security/ civilian-drones-fixing-an-image-problem. Accessed on: 2020-12-10.

[13] M. Erdelj and E. Natalizio, "UAV-assisted disaster management: Applications and open issues". In: 2016 International Conference on Computing, Networking and Communications (ICNC), 2016, 10.1109/ICCNC.2016.7440563.

[14] A. C. Watts, V. G. Ambrosia and E. A. Hinkley, "Unmanned Aircraft Systems in Remote Sensing and Scientific Research: Classification and Considerations of Use", Remote Sensing, vol. 4, no. 6, 2012, 1671-1692, $10.3390 /$ rs4061671.

[15] S. G. Gupta, M. M. Ghonge and P. M. Jawandhiya, "Review of Unmanned Aircraft System (UAS)", International Journal of Advanced Research in Computer Engineering \& Technology (IJARCET), vol. 2, no. 4, 2013, 1646-1658.

[16] M. Hassanalian and A. Abdelkefi, "Classifications, applications, and design challenges of drones: A review", Progress in Aerospace Sciences, vol. 91, 2017, 99-131, 10.1016/j.paerosci.2017.04.003.

[17] I. Korobiichuk, V. Karachun and V. Mel'nick, "Stochastic Structure of Inciting Factors of Trivial Gyrostabilized Platform". In: R. Szewczyk, J. Krejsa, M. Nowicki and A. OstaszewskaLiżewska (eds.), Mechatronics 2019: Recent Advances Towards Industry 4.0, 2020, 36-44, 10.1007/978-3-030-29993-4_5.

[18] I. Korobiichuk, "Experimental Investigations of a Precision Sensor for an Automatic Weapons Stabilizer System", Sensors, vol. 17, no. 1, 2017, 10.3390/s17010023.

[19] T. A. Johansen, "Stability, robustness, and performance of fuzzy model based control". In: Proceedings of $35^{\text {th }}$ IEEE Conference on Decision and Control, 1996, 604-609, 10.1109/CDC.1996.574390.

[20] K. M. Passino and S. Yurkovich, Fuzzy Control, Addison-Wesley, 1997.

[21] V. Tregub, I. Korobiichuk, O. Klymenko, A. Byrchenko and K. Rzeplińska-Rykała, "Neural Network Control Systems for Objects of Periodic Action with Non-linear Time Programs". In: R. Szewczyk, C. Zieliński and M. Kaliczyńska (eds.), Automation 2019, 2020, 155-164, 10.1007/978-3-030-13273-6_16.

[22] I. Korobiichuk, V. Tregub, O. Klymenko, I. Elperin, V. Sidletskyi, Y. Smityuh and M. Chorno- van, "Development of Logical Control System for the Purification Department at Molasses Production". In: R. Szewczyk, J. Krejsa, M. Nowicki and A. Ostaszewska-Liżewska (eds.), Mechatronics 2019: Recent Advances Towards Industry 4.0, 2020, 206-213, 10.1007/978-3-030-29993-4_26.

[23] “Наукові розробки (in Ukrainian)”. National Aviation University, https://nau.edu.ua/ua/ menu/science/naukovi-rozrobki/. Accessed on: 2020-12-10. 